\title{
Vibrações mecânicas: Um agente estressor no transporte de pintos
}

\author{
Ana C. Donofre ${ }^{1}$, Iran J. O. da Silva² \& Aérica C. Nazareno ${ }^{3}$ \\ ${ }^{1}$ Departamento de Engenharia de Biossistemas, NUPEA/ESALQ/USP, Piracicaba, SP. E-mail: acdonofre@gmail.com (Autor correspondente) \\ ${ }^{2}$ Departamento de Engenharia de Biossistemas, NUPEA/ESALQ/USP, Piracicaba, SP. E-mail: iranoliveira@usp.br \\ ${ }^{3}$ Departamento de Engenharia de Biossistemas, NUPEA/ESALQ/USP, Piracicaba, SP. E-mail: aericacn@yahoo.com.br
}

\section{Palavras-chave:}

avicultura

aceleração

frequência respiratória

\begin{abstract}
R E S U M O
As vibrações mecânicas, presentes no transporte de cargas vivas, podem comprometer a estabilidade fisiológica e o futuro desempenho dos animais de produção. O objetivo desta pesquisa foi avaliar, por meio de simulações, o efeito de dois níveis de aceleração $\left(9,64\right.$ e 15,19 $\left.\mathrm{m} \mathrm{s}^{-2}\right)$ sobre o estresse e o desempenho de pintos de corte de um dia. As vibrações foram simuladas utilizando-se um agitador mecânico e as intensidades de vibração estudadas por valores de aceleração geral. O período de simulação durou duas horas para cada tratamento e as aves tiveram seus parâmetros (diferença de peso; frequência respiratória e desempenho na primeira semana) comparados a grupos-controle (sem vibração). Realizou-se um delineamento inteiramente casualizado em que os resultados obtidos indicaram que esses níveis não influenciaram significativamente $(\mathrm{p}<0,05)$ na diferença de peso nem no desempenho na primeira semana de vida; entretanto, os pintos submetidos à aceleração de $15,19 \mathrm{~m} \mathrm{~s}^{-2}$ obtiveram um aumento significativo $(\mathrm{p}<0,05)$ na frequência respiratória $\left(54,33 \mathrm{mov} \mathrm{min}^{-1}\right)$ concluindo-se, assim, que as vibrações podem atuar como potencial estressor no transporte dessas aves.
\end{abstract}

\section{Key words:} poultry production acceleration respiratory frequency

\section{Mechanical vibrations: A stressor in the transport of chicken}

\begin{abstract}
A B S T R A C T
Mechanical vibrations present in transporting live loads can impair physiologic stability and the future performance of livestock. The aim of this study was to evaluate, using simulations, the effect of two levels of vibration (9.64 and $15.19 \mathrm{~m} \mathrm{~s}^{-2}$ ) in stress responses and performance of day-old chicken. The vibrations were simulated by means of a shaking machine and studied by general acceleration values. The simulation period lasted two hours for each treatment and the birds had their parameters (difference in weight, respiratory rate, and performance in the first week) compared to control groups (without vibration). The experiment was conducted in a randomized design and the results showed that these levels did not differ significantly $(\mathrm{p}<0.05)$ in weight and performance in the first week of life. However, chicks subjected to acceleration of $15.19 \mathrm{~m} \mathrm{~s}^{-2}$ achieved a significant increase $(\mathrm{p}<0.05)$ in respiratory rate $(54.33 \mathrm{mov}$ $\mathrm{min}^{-1}$ ), thus concluding that the vibrations may act as a potential stressor during transport of these birds.
\end{abstract}

\section{INTRODUÇÃO}

O transporte é um componente essencial na indústria avícola moderna por ser responsável pela comunicação entre os diversos segmentos produtivos como, por exemplo, entre os incubatórios e as granjas de criação, com a distribuição de pintos de um dia. Todavia e dependendo da magnitude dos desafios impostos, o transporte gera impactos que podem comprometer a saúde, o bem-estar e o desempenho produtivo das aves (Mitchell \& Kettlewell, 2009; Schwartzkopf-Genswein et al., 2012).

Abeyesinghe et al. (2001), Ljungberg et al. (2007), Mitchell \& Kettlewell (2009), Silva \& Vieira (2010) e SchwartzkopfGenswein et al. (2012) relatam que, quando transportados, frangos de corte são expostos a uma gama de fatores que atuam de forma isolada ou em combinação resultando em situações de estresse e desconforto. Dentre esses fatores as condições das estradas e do veículo, da mesma forma que o desempenho do motorista durante a condução, são determinantes na ocorrência de um evento ainda pouco estudado, ou seja, as vibrações mecânicas (Carlisle et al., 1998; Walber \& Tamagna, 2010; Gebresenbet et al., 2011; Nazareno, 2012).

As vibrações apresentam efeitos prejudiciais sobre o veículo por completo (chassi, suspensão, eixos, rodas e pneus) os quais são transmitidos para a carga carregada ocasionando diferentes respostas e consequências que, em sua maioria, são indesejáveis (Bovenzi, 2005; Sotelo, 2006; Ranathunga et al., 2010). A ocorrência de vibrações resulta em falhas estruturais e mecânicas em equipamentos enquanto em seres vivos este efeito está relacionado a situações de dor e desconforto e, ainda, à redução da eficiência dos sistemas biológicos (Rao, 2008).

Segundo Randall et al. (1997) as faixas de aceleração são consideradas pouco aversivas quando se encontram de 0,315 a $0,63 \mathrm{~m} \mathrm{~s}^{-2}$, aversivas de $0,5 \mathrm{a} 1,6 \mathrm{~m} \mathrm{~s}^{-2}$ e extremamente aversivas se forem superiores a $4,0 \mathrm{~m} \mathrm{~s}^{-2}$. Em estudos realizados por meio de testes de escolha observou-se que frangos adultos tendem a evitar vibrações com acelerações de $10 \mathrm{~m} \mathrm{~s}^{-2}$, comparadas às acelerações de $5 \mathrm{~m} \mathrm{~s}^{-2}$ (Rutter \& Randall, 1993). 
Gebresenbet et al. (2011) propuseram faixas ideais de aceleração de 0,5 a $1,15 \mathrm{~m} \mathrm{~s}^{-2}$ entretanto, no transporte em estradas brasileiras já foram observados valores médios de aceleração geral superiores a 10,4 $\mathrm{m} \mathrm{s}^{-2}$ (Nazareno, 2012).

Segundo Carlisle et al. (1998) e Minka \& Ayo (2009) as vibrações durante o transporte podem contribuir potencialmente para a fadiga e incidência de lesões nas aves e, assim, induzir a respostas fisiológicas resultantes do estresse experimentado pelos animais. Durante exposições de $3 \mathrm{~h}$ a vibrações mecânicas observou-se um aumento na temperatura corporal e redução no pH muscular de frangos de corte (Warris et al., 1997).

Abeysingne et al. (2001) verificaram que um período de 60 min de exposição aos efeitos da vibração é suficiente para provocar estresse em frangos; além disto, acelerações superiores a $2 \mathrm{~m} \mathrm{~s}^{-2}$ resultam na queda significativa dos níveis de glicose e $\mathrm{pH}$ do peito e das coxas de frangos de corte. Tais resultados refletem o esgotamento das reservas de energia resultante do estresse causado pela exposição às vibrações (Warris et al.,1997; Carlisle et al., 1998).

Sabe-se que o transporte das aves recém-nascidas exige cuidados específicos, muitas vezes mais intensos do que os impostos no transporte de frangos adultos. Vieira (2011) avaliou os efeitos das condições simuladas de transporte de pintos de um dia e encontrou que tais condições influenciaram nas respostas fisiológicas e produtivas dos animais. Segundo o autor, em situações de campo pouca padronização é observada sujeitando as aves a diversos fatores estressantes que contribuem de forma conjunta na desuniformidade do lote e em perdas, antes mesmo do alojamento.

Apesar dos estudos já realizados ainda se nota a necessidade de mais pesquisas e diferentes meios de medição dos efeitos da vibração em aves, sobretudo em etapas ainda pouco estudadas, como o transporte de pintos dos incubatórios para as granjas de criação. Considerando que os efeitos das vibrações não foram até então caracterizados para essa categoria da indústria avícola, o objetivo da pesquisa foi avaliar, por meio de simulações, o efeito de dois tratamentos de vibração sobre o estresse e o desempenho de pintos de corte de um dia.

\section{Material e Métodos}

O ensaio foi realizado em 2011, no laboratório do Núcleo de Pesquisa em Ambiência (NUPEA) da Escola Superior de Agricultura "Luiz de Queiroz" (ESALQ/USP), Piracicaba, SP. Para as simulações utilizou-se uma mesa agitadora capaz de reproduzir os movimentos das vibrações mecânicas nos três eixos ortogonais ( $x, y$ e $z$ ) em diferentes faixas de atuação.

Foram testados dois níveis de aceleração geral (RSS; $\mathrm{m} \mathrm{s}^{-2}$ ) que caracterizaram os tratamentos de vibração. Tais valores representaram um nível inferior de $9,64 \mathrm{~m} \mathrm{~s}^{-2}\left(\mathrm{~V}_{1}\right)$, próximo a valores encontrados por Nazareno (2012) em condições reais de transporte e um nível superior de $15,19 \mathrm{~m} \mathrm{~s}^{-2}\left(\mathrm{~V}_{2}\right)$ alcançado pela mesa vibratória.

Para caracterizar os tratamentos de vibração utilizouse de dois acelerômetros triaxiais $\mathrm{HOBO}$ pendant $\mathrm{G}^{\circ}$, os quais possuíam faixa de medição de $\pm 3 \mathrm{~g}$ ou $29,4 \mathrm{~m} \mathrm{~s}^{-2}$. Os acelerômetros foram acoplados horizontalmente a $90^{\circ} \mathrm{em}$ duas extremidades opostas das caixas de transporte, em um espaço vago das mesmas para que a movimentação das aves não tirasse o equipamento de sua posição (Figura 1).

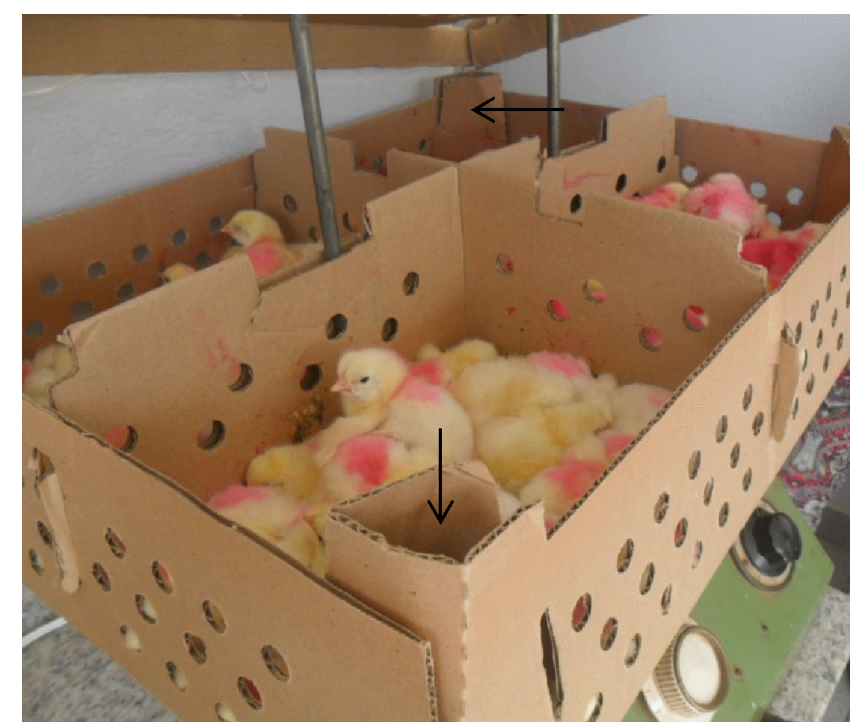

Figura 1. Simulação de um tratamento de vibração. As flechas indicam o local de posicionamento dos acelerômetros

Os acelerômetros foram programados com o software HOBOWARE ${ }^{\oplus} \mathrm{RO}$, para coleta dos valores de aceleração instantânea nos três eixos ortogonais ( $\mathrm{x}, \mathrm{y}$ e $\mathrm{z}$ ), em intervalos de dez segundos a uma frequência de $2 \mathrm{~Hz}$. Para obter o valor de aceleração geral (RSS; $\mathrm{m} \mathrm{s}^{-2}$ ), foi calculada a raiz quadrática média (RMS; $\mathrm{m} \mathrm{s}^{-2}$ ) dos três eixos adotando-se as equações aplicadas nos estudos de Garcia et al. (2008), Gebresenbet et al. (2011) e Nazareno (2012).

A RMS (Eq. 1) é dada pela raiz quadrática média dos valores de aceleração instantânea ocorridos em determinado período de medição enquanto a RSS (Eq. 2), por meio da raiz quadrada do somatório dos quadrados da RMS nos eixos x, y e z, ou seja,

$$
\operatorname{RMS}_{\mathrm{j}}=\left(\frac{\Sigma_{\mathrm{t}} \mathrm{a}(\mathrm{t})_{\mathrm{j}}^{2}}{\mathrm{~N}}\right)^{1 / 2}
$$

em que $a_{j}(t)$ é o valor observado da aceleração instantânea no eixo $\mathrm{j}(\mathrm{x}, \mathrm{y}$ ou $\mathrm{z})$ e no tempo $\mathrm{t}(\mathrm{t}=1,2, \ldots, \mathrm{N})$, sendo $\mathrm{N}$ o número total de observações no respectivo eixo, e

$$
\mathrm{RSS}=\left(\mathrm{RMS}_{\mathrm{x}}^{2}+\mathrm{RMS}_{\mathrm{y}}^{2}+\mathrm{RMS}_{\mathrm{z}}^{2}\right)^{1 / 2}
$$

em que RMSx é o valor da raiz média quadrática do eixo $\mathrm{x}$ (vertical), RMSy é o valor da raiz média quadrática do eixo y (horizontal, da direita para a esquerda) e RMSz é o valor da raiz média quadrática do eixo $\mathrm{z}$ (horizontal de frente para trás).

Os valores da raiz média quadrática (RMS; $\mathrm{m} \mathrm{s}^{-2}$ ) dos três eixos e da RMS resultante (RSS; $\mathrm{m} \mathrm{s}^{-2}$ ) que caracterizaram os dois tratamentos avaliados são apresentados na Tabela 1. 
Tabela 1. Valores de aceleração média e geral, RMS e RSS $\left(\mathrm{m} \mathrm{s}^{-2}\right)$, para os níveis de aceleração $\left(\mathrm{V}_{1}\right.$ e $\left.\mathrm{V}_{2}\right)$ que caracterizaram as vibrações mecânicas

\begin{tabular}{ccc}
\hline \multirow{2}{*}{ Aceleração } & \multicolumn{2}{c}{ Tratamentos de vibração $\left(\mathbf{m ~ s}^{\mathbf{2}}\right)$} \\
\cline { 2 - 3 } RMSx & $\mathbf{V}_{\mathbf{1}}$ & $\mathbf{V}_{\mathbf{2}}$ \\
RMSy & 7,60 & 9,74 \\
RMSz & 4,49 & 7,54 \\
RSS & 3,86 & 8,89 \\
& 9,64 & 15,19 \\
\hline
\end{tabular}

Cada tratamento teve seu grupo controle sem vibração $\left(\mathrm{C}_{1}\right.$ e $\mathrm{C}_{2}$ ) para comparação visto que as caixas referentes aos níveis foram colocadas separadamente no equipamento e submetidas a um período de exposição de duas horas interruptas. Foram totalizados, assim, quatro grupos experimentais, dois expostos às vibrações $\left(V_{1} e V_{2}\right)$ e dois não expostos às vibrações, ou seja, os tratamentos controle $\left(\mathrm{C}_{1}\right.$ e $\left.\mathrm{C}_{2}\right)$ de cada tratamento de vibração.

Foram utilizadas 400 aves com um dia de idade da linhagem Cobb - 500, de ambos os sexos e já vacinadas, provenientes de um mesmo incubatório. As aves, recebidas simultaneamente, foram misturadas e redistribuídas ao acaso em caixas de transporte específicas para pintos de um dia, construída em cartão canelado ( $570 \times 473 \times 125 \mathrm{~mm}$ ), com quatro divisões, em que cada uma tinha capacidade para 25 aves, totalizando 100 pintos por caixa.

Foram selecionadas ao acaso doze aves de cada divisão das caixas dos tratamentos. Essas aves foram identificadas com anilhas para a coleta das variáveis respostas relacionadas com o peso, o que resultou no total de 48 aves identificadas por tratamento. Ao término do processo de identificação as aves permaneceram em descanso durante uma hora, antes das simulações, a fim de amenizar os supostos efeitos do estresse da manipulação inicial.

Após o período de descanso um dos tratamentos de vibração foi sorteado para o início das simulações; ao término da exposição, por duas horas aos efeitos das vibrações no tratamento em questão obtiveram-se as respostas frequência respiratória (FR) e perda de peso durante a exposição (DP).

A frequência respiratória foi obtida imediatamente após o desligamento da máquina. Para que não houvesse contato da ave com o avaliador a tomada dessa resposta foi feita com os pintos ainda dentro da caixa pela contagem visual dos movimentos abdominais durante $15 \mathrm{~s}$; em seguida, multiplicou-se o valor obtido por 4 para a estimativa de $1 \mathrm{~min}$. Observaram-se cinco aves ao acaso para cada uma das divisões da caixa de transporte, totalizando 20 aves por tratamento.

Para o cálculo da DP utilizaram-se todas as aves anilhadas inicialmente, as quais foram pesadas, uma a uma, em balança semianalítica, antes das simulações e depois da avaliação da FR. Com a diferença das duas medidas de massa para cada ave obteve-se a resposta DP. Procedeu-se à tomada dos parâmetros fisiológicos alternando as aves expostas às vibrações com as do grupo sem vibração, controle referente àquele nível; logo após a primeira simulação os mesmos procedimentos foram adotados para o segundo tratamento de vibração e para seu grupo controle; posteriormente, as 400 aves foram alojadas e mantidas durante uma semana em um círculo de proteção com condições ambientais controladas e semelhantes às de uma granja de criação (aquecimento e ração inicial à vontade) período no qual se registrou o peso diário das aves anilhadas no início para se estimar o peso final $(\mathrm{PF})$ de cada grupo $\left(\mathrm{V}_{1}, \mathrm{~V}_{2}, \mathrm{C}_{1}\right.$ $\mathrm{e}_{2}$ ) na primeira semana de criação; além disto contabilizou-se, dentro dos tratamentos, o número de aves mortas.

$\mathrm{O}$ delineamento experimental utilizado foi inteiramente casualizado. Foram consideradas repetições as aves anilhadas de cada divisão das caixas. Realizou-se a análise da distribuição das respostas de interesse que constituíam a frequência respiratória (FR), diferença de peso (DP) e peso ao final de sete dias (PF) em cada um dos grupos.

As médias de tratamento foram comparadas com as dos seus grupos controles a partir do teste t unilateral considerando-se o nível de significância de 5\%. A pressuposição de normalidade foi avaliada com o teste de Shapiro-Wilk e a de homogeneidade de variância por meio do teste de Bartlett. A qualidade do ajuste dos modelos de análise de variância para as respostas FR, DP e PF foi avaliada por meio de gráfico dos resíduos e envelopes simulados; enfim, todos os procedimentos foram realizados utilizando-se o programa estatístico R.

\section{Resultados e Discussão}

Na Tabela 2 se observa que os efeitos dos tratamentos de vibração, $V_{1}\left(9,64 \mathrm{~m} \mathrm{~s}^{-2}\right)$ e $V_{2}\left(15,64 \mathrm{~m} \mathrm{~s}^{-2}\right)$, não foram significativos para a resposta diferença de peso (DP) a nível de 0,05 de significância, sendo as médias dos tratamentos expostos às vibrações próximas às de seus controles.

$O$ fato de não serem encontrados resultados significativos para a diferença de peso, corrobora com os resultados de Garcia et al. (2008), os quais submeteram frangos adultos a dois níveis de vibração simulada, com RSS de 8,7 e $22,2 \mathrm{~m} \mathrm{~s}^{-2}$, associados a três tempos de exposição (30, 60 e $90 \mathrm{~min})$ e também não verificaram influência da exposição às vibrações sobre esta resposta. Em condições simuladas de transporte com diferentes faixas térmicas o peso dos pintos de um dia também não foi alterado (Vieira et al., 2012). Tal resultado, apesar de não estar associado às vibrações mecânicas, evidencia que o peso das aves é pouco influenciado pelo estresse durante o transporte e que outras medidas devem ser adotadas.

Randall et al. (1993) sugerem que as alterações fisiológicas e psicológicas nas aves expostas às vibrações podem resultar

Tabela 2. Médias e valores do teste t unilateral, considerando-se $5 \%$ de significância obtidos para as respostas diferença de peso (g), frequência respiratória $\left(\right.$ mov $\left.\mathrm{min}^{-1}\right)$ e peso ao final da primeira semana (g) nos tratamentos $\mathrm{V}_{1}$ e $\mathrm{V}_{2}$ comparados aos seus respectivos tratamentos controle, $\mathrm{C}_{1}$ e $\mathrm{C}_{2}$

\begin{tabular}{|c|c|c|c|c|c|c|}
\hline \multirow{2}{*}{ Resposta } & \multicolumn{6}{|c|}{ Tratamento } \\
\hline & $V_{1}$ & $\mathbf{C}_{1}$ & $p$ & $V_{2}$ & $\mathbf{C}_{2}$ & $p$ \\
\hline DP & $-0,29$ & $-0,21$ & 0,1292 & $-0,38$ & $-0,19$ & 0,0764 \\
\hline FR & 47 & 40 & 0,0634 & 54,33 & 42,67 & 0,0026 \\
\hline PF & 111,97 & 112,80 & 0,4261 & 112,42 & 107,70 & 0,9047 \\
\hline
\end{tabular}

DP - Diferença de peso; FR - Frequência respiratória; PF - Peso ao final da primeira semana 
em sintomas semelhantes aos que ocorrem em seres humanos, quando submetidos aos mesmos efeitos. Segundo este autor, entre os sintomas se destacam o medo, alterações na frequência respiratória e fadiga muscular, resultantes da distribuição dos movimentos oscilatórios e forças dentro do corpo.

A frequência respiratória (FR) é um parâmetro fisiológico extremamente relevante no diagnóstico do estado da saúde do animal, (faltou vírgula) imediata em condições de estresse como o transporte (Minka \& Ayo, 2009). Como resposta ao estresse verificou-se, neste estudo, que em média os dois tratamentos submetidos a vibração, apresentaram um aumento na FR quando comparados aos seus controles; todavia, para a menor aceleração, $V_{1}\left(9,64 \mathrm{~m} \mathrm{~s}^{-2}\right)$, não se verificou diferença estatística $(\mathrm{p}<0,05)$ entre o tratamento e o seu grupo controle, apesar do valor $\mathrm{p}$ obtido ser baixo $(0,0634)$.

$\mathrm{O}$ tratamento de vibração mais intenso $\left(\mathrm{V}_{2}=15,64 \mathrm{~m} \mathrm{~s}^{-2}\right)$ resultou em 54,33 mov $\mathrm{min}^{-1}$ e seu controle $\left(C_{2}\right)$ em 42,67 mov $\min ^{-1}$. Este aumento foi significativo $(\mathrm{p}=0,00261)$ pelo teste $\mathrm{t}$ unilateral $(\mathrm{p}<0,05)$ confirmando um provável comprometimento das funções fisiológicas das aves expostas às vibrações durante o transporte. Segundo Marchini et al. (2007) e Nascimento et al. (2012), a frequência respiratória média de frangos na primeira semana de vida em situação de conforto, é em torno de 48 mov min $^{-1}$.

Nos grupos controles verificou-se que as médias de FR foram próximas entre si $\left(\mathrm{C}_{1}=40,67 \mathrm{mov} \cdot \mathrm{min}^{-1} \mathrm{e}_{2}=42,67 \mathrm{mov} \mathrm{min}^{-1}\right)$. Vieira et al. (2012) verificaram, em simulações do transporte de pintos de um dia, variações nas variáveis fisiológicas das aves após 3 horas de exposição em relação ao seu tratamento controle, em que a FR foi significativamente maior $(\mathrm{P}<0,05)$ nos tratamentos estressados no transporte.

É de conhecimento geral que a transmissão das vibrações do piso do veículo para um corpo pode criar condições desconfortáveis por meio do deslocamento do centro de gravidade do indivíduo, o que resulta em perturbações no organismo e situações de mal-estar (Randall, 1992; Carlisle et al., 1998). Ainda são escassos os estudos relativos aos efeitos das vibrações em aves; entretanto, não se pode afirmar que tal evento não induza ao estresse durante o transporte. Já se observou, por meio de testes de escolha, que frangos adultos evitam as vibrações quando elas estão agindo a maiores valores de aceleração (Rutter \& Randall, 1993; Abeyesinghe et al., 2001).

A evolução do desempenho na primeira semana de vida pode ser verificada na Figura 2. Nota-se que entre os quatro grupos $\left(V_{1}, V_{2}, C_{1}\right.$ e $\left.C_{2}\right)$ o peso mostrou-se bastante homogêneo ao longo do período de observação.

As médias do peso final (PF) dos tratamentos se mantiveram próximas entre si $\left(\mathrm{V}_{1}=111,97 \mathrm{~g} ; \mathrm{C}_{1}=112,8 \mathrm{~g}, \mathrm{~V}_{2}=112,42\right.$ $\mathrm{g} \mathrm{e}_{2}=107,07 \mathrm{~g}$ ) e pelo teste $\mathrm{t}$ não se verificou diferença significativa $(\mathrm{p}>0,05)$ nas comparações dos tratamentos vibrados com os seus respectivos grupos controles. Acredita-se que, apesar do estresse momentâneo apontado pelo aumento na frequência respiratória das aves, a exposição às vibrações mecânicas não venha interferir na uniformidade nem no seu futuro desempenho. Foram contabilizadas 12 mortes entre $\mathrm{V}_{1}$, $\mathrm{V}_{2}, \mathrm{C}_{1}$ e $\mathrm{C}_{2}$ na primeira semana de criação, seis das quais foram

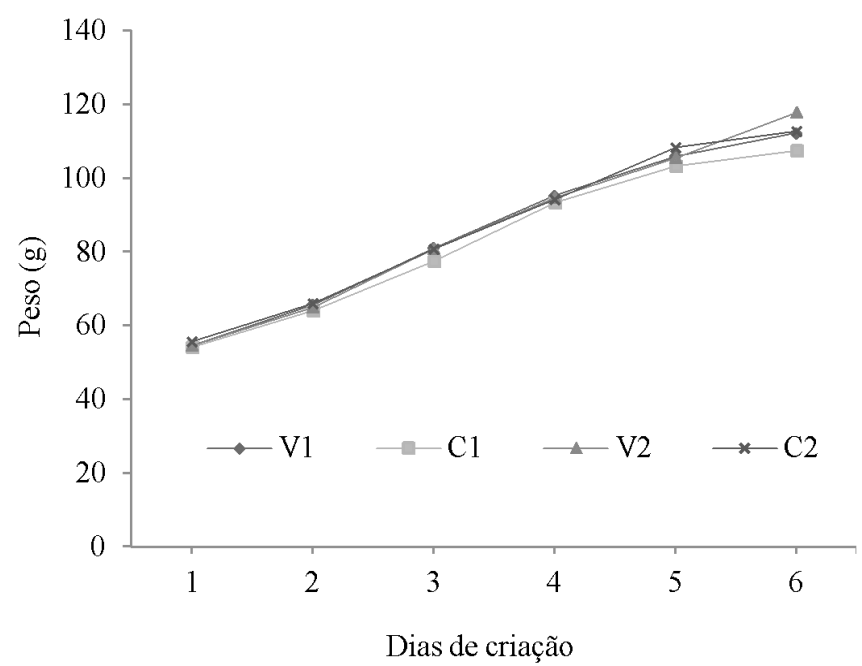

Figura 2. Variação do peso ao longo dos dias de criação para os tratamentos de vibração $V_{1}(9,64$ $\left.\mathrm{m} \mathrm{s}^{-2}\right)$ e $\mathrm{V}_{2}\left(15,19 \mathrm{~m} \mathrm{~s}^{-2}\right)$, e controles $\mathrm{C}_{1}$ e $\mathrm{C}_{2}$

referentes ao tratamento submetido à maior aceleração $(15,19$ $\mathrm{m} \mathrm{s}^{-2}$ ) e as demais distribuídas igualmente entre os outros tratamentos. Porém não se pôde afirmar que o ocorrido se deu em virtude da exposição à vibração visto que esses registros não são informativos a este ponto e baixos valores de mortalidades são admissíveis para a primeira semana de vida das aves.

\section{Conclusões}

1. Não foram comprovadas perdas de peso antes nem após a submissão ao efeito da vibração; tampouco houve queda de desempenho na primeira semana de alojamento e mortalidades em função de tal efeito.

2. Os pintos submetidos ao maior valor de aceleração apresentaram um aumento significativo em sua frequência respiratória.

3. As vibrações mecânicas podem ser consideradas UM fator estressante para as aves durante o transporte.

\section{Literatura Citada}

Abeyesinghe, S. M.;Wathes, C. M.; Nicol, C. J.; Randall, J. M. The aversion of broiler chickens to concurrent vibrational and thermal stressors. Applied Animal Behaviour Science, v.73, p.199-215, 2001.

Bovenzi, M. Health effects of mechanical vibration. Giornale Italiano di Medicina del Lavoro ed Ergonomia v.27, p.5864, 2005.

Carlisle, A. J.; Mitchell, M. A.; Hunter, R. R.; Duggan, J. A.; Randall, J. M. Physiological responses of broiler chickens to the vibrations experienced during road transportation. British Poultry Science, v.39, p.48-49, 1998.

Garcia, D. B.; Silva, I. J. O.; Barbosa Filho, J. A. S.; Vieira, F. M. C; Dias, C. T. S. Evaluation of the effect of vibration in simulated condition of transport of broiler chickens. In: International Livestock Environment Symposium, 2008. Foz do Iguaçu. Anais...Foz de Iguaçu: CIRG, 2008. CD-Rom 
Gebresenbet, G.; Aradom, S.; Bulitta, F. S.; Hjerpe, E. Vibration levels and frequencies on vehicle and animals during transport. Biosystems Engineering, v.110, p.10$19,2011$.

Ljungberg, D.; Gebresenbet, G.; Aradom, S. Logistics chain of animal transport and abattoir operations. Biosystems Engineering, v.96, p.267-277, 2007.

Marchini, C. F. P.; Silva, P. L. Nascimento, M. R. B. M.; Tavaver, $M$. Frequência respiratória e temperatura cloacal em frangos de corte submetidos à temperatura ambiente cíclica elevada. Archives of Veterinary Science, v.12, p.41-46, 2007.

Minka, N. S.; Ayo, J. O. Physiological responses of food animals to road transportation stress - Review. African Journal of Biotechnology, v.8, p.7415-7427, 2009.

Mitchell, M. A.; Kettlewell, P. J. Welfare of poultry during transport - a review. In: Poultry Welfare Symposium, 2009, Cervia. Worlds poultry science. Cervia: Association Proceeding, 8 th. 2009. p.90-100.

Nascimento, S. T.; Silva, I. J. O.; Mourão, G. B.; Castro, A. C. Bands of respiratory rate and cloacal temperature for different broiler chicken strains. Revista Brasileira de Zootecnia, v.41, p.1318-1324, 2012.

Nazareno, A. C. Ambiência pré-porteira: Avaliação das condições bioclimáticas e das operações pré-eclosão na qualidade de pintos de corte. Piracicaba: ESALQ/USP, 2012. 208p. Tese Doutorado

Ranathunga, C. L.; Jayaweera, H. H. E.; Suraweera, S. K. K.; Wattage, S. C.; Ruvinda, K. K. D. L.; Ariyaratne, T. R. Vibration effects in vehicular road transportation. Proceedings of the Technical Sessions, v.26, p.9-16, 2010.

Randall, J. M. Human subjective response to lorry vibration: Implications for farm animal transport. Journal of Agricultural Engineering Research, v.52, p.295-307, 1992.

Randall, J. M.; Duggan, J. A.; Alami, M. A.; White, R. P. Frequency weightings for the aversion of broiler chickens to horizontal and vertical vibration. Journal of Agricultural Engineering Research, v.68, p.387-397, 1997.
Randall, J. M.; Randall, J. M.; Streader, W. V.; Meehan, A. M. Vibration on poultry transporters. Britsh Poultry Science, v.34, p.635-642, 1993.

Rao, S. S. Vibrações mecânicas. 4.ed. São Paulo: Pearson Prentice Hall, 2008.424p.

Rutter, S. M.; Randall, J. M. A version of domestic fowl to whole body vibratory motion. Applied Animal Behaviour Science, v.37, p.69-73, 1993.

Schwartzkopf-Genswein, K. S.; Faucitano, L. S.; Dadgar, P.; Shand, L. A.; González, T. G. Road transport of cattle, swine and poultry in North America and its impact on animal welfare, carcass and meat quality: A review. Meat Science, v.92, p.227-243, 2012.

Silva, I. J. O.; Vieira, F. M. C. Ambiência animal e as perdas produtivas no manejo pré-abate: $\mathrm{O}$ caso da avicultura de corte brasileira. Archivos de Zootecnia, v.59, p.113-131, 2010.

Sotelo, J. J. Introdução às vibrações mecânicas. São Paulo: Edgar Blucher, 1.ed., 2006. 168p.

Vieira, F. M. C. Transporte Animal: Influência das condições bioclimáticas no desempenho produtivo e fisiológico de pintos de um dia. Piracicaba: ESALQ/USP, 2011, 87p. Tese Doutorado

Vieira, F. M. C.; Silva, I. J. O.; Miranda, K. O. S.; Nazareno, A. C.; Camargo, J. R.; Vieira, A. M. C. Thermoregulatory responses of day-old chickens submitted to simulated transport condition: Effect of exposure time under different thermal ranges. In: International Livestock Environment Symposium, 9, 2012, Valencia. Proceedings...Valencia: ASABE Conference Presentation, 2012. CD-Rom

Walber, M.; Tamagna, A. Avaliação dos níveis de vibração existentes em passageiros de ônibus rodoviários intermunicipais, análise e modificação projetual. Revista Liberato, v.11, p.81-88, 2010.

Warriss, P. D.; Brown, S. N.; Knowles, T. G.; Edwards, J. E.; Duggan, J. A. Potential effect of vibration during transport on glycogen reserves in broiler chickens. The Veterinary Journal, v.153, p.215-219, 1997. 